\title{
Single Operation with Simplified Incisions to Build an Experimental Cerebral Aneurysm Model by Induced Hemodynamic Stress and Estrogen Deficiency in Rats
}

\author{
Cong WU1', Yi LIU1', Min HE¹, Lei ZHU², Chao YOU¹ \\ 'Sichuan University, West China Hospital, Department of Neurosurgery, Chengdu, Sichuan Province, China \\ ${ }^{2}$ Sichuan University, Department of Human Anatomy, Chengdu, Sichuan Province, China
}

\section{ABSTRACT}

AIM: To implement a surgical technique consisting of simplified incisions that allows all required procedures to be performed in one single operation for the purpose of reducing surgical stress in experimental animals.

MATERIAL and METHODS: Experimental animals (rats) were assigned to one of four groups: Group 0 was the (normal) control group, Group 1 consisted of rats that had an operation using multiple incisions, Group 2 consisted of rats who received a midline incision and were raised for 3 months, and Group 3 consisted of rats who also received a midline incision, but had been raised for 6 months. Rat blood pressure was measured by tail cuff method. The surgical characteristics and outcomes of the rats in Groups 1 and 2 were compared. Aneurysmal lesions of both branching and non-branching sites were compared amongst the 4 groups by observation of the cerebral vascular corrosion casts through a scanning electron microscope. Histological analyses of the induced aneurysms were performed.

RESULTS: The simplified incision technique significantly reduced the length of surgery and need for anesthesia redose during the operation. No aneurysms formed in the normal control rats. The incidence of saccular aneurysm formation significantly increased in Group 3. Histological analyses confirmed the aneurysms induced in the rats shared the same characteristics as human aneurysms.

CONCLUSION: Our modified surgical method reduced the surgical stress in rats. It also successfully induced both saccular and fusiform cerebral aneurysms. While longer incubation duration for aneurysm formation could be applied in future researches.

KEYWORDS: Cerebral aneurysm, Animal model, Oophorectomy, Etiology, Rats

\section{INTRODUCTION}

Aneurysmal subarachnoid hemorrhage is a significant cause of morbidity and mortality throughout the world and it continues to present challenging problems despite the advances in neurosurgery field (2). Various animal models have been developed to reveal the underlying mechanisms and possible treatments of Cerebral Aneurysm $(C A)(5,6,18)$. A widely used experimental CA rat model, reported by Hashimoto et al, consists of induction of an aneurysm secondary to hypertension resulting from the bilateral ligation of the posterior renal arter- ies and unilateral ligation of common carotid artery to enhance the hemodynamic stress placed on the collateral side of the Willis circle (5). Since estrogen deficiency has been showed to increase the frequency of aneurysm formation, a bilateral oophorectomy was performed one month after ligation of the arteries, as a second (two-stage) operation $(7,8)$.

Recently, an experimental CA rat model consisting of a one stage operation involving ligation of the unilateral common carotid artery, ligation of the bilateral posterior renal arteries, and oophorectomy has been reported. However, the authors 
used two incisions: a midline abdominal incision to perform the oophorectomy and bilateral flank incisions to ligate the renal arteries (3). Therefore, to reduce the stress placed on the experimental animals and potential surgical complications related to a two-step operation and/or multiple incisions, we designed a one-step operation involving a single midline dorsal incision that allowed both the ligation of bilateral renal arteries and oophorectomy to be performed concurrently. Surgical characteristics and outcomes were compared between rats that received the multiple vs. simplified incisions. Additionally, the rate of aneurysm formation was compared between the 3 months and 6 months aneurysm incubation groups.

\section{MATERIAL and METHODS}

This experiment protocol has been approved by the animal ethics committee of Sichuan University. Seven weeks old female Sprague-Dawley rats were used. The rats were assigned to one of four groups. Group 0 was the normal control group, and consisted of rats that had been raised for 3 months without any intervention. Group 1 consisted of rats that had received multiple incisions. Rats in Group 2 and 3 received one simplified midline incision; rats in Group 2 had been raised for 3 months, while Group 3 had been raised for 6 months.

The previously used multiple incision surgical procedure consisted of three incisions: a midline abdominal incision to remove both ovaries, a bilateral flank incision to access renal arteries, and a neck incision to ligate the right common carotid artery.

The simplified incision procedure that was performed on rats in Group 2 and 3 is described as follows. After proper anesthesia was administered by use of intraperitoneal injections of ketamine $(80 \mathrm{mg} / \mathrm{kg})$ and xylazine $(8 \mathrm{mg} / \mathrm{kg})$, the operational area was shaved and rats were placed in the prone position. The skin incision was designed on the middle line under the inferior line of costal margin (Figure 1A). Subcutaneous fascia was cut and bluntly separated; this was a key method to allow the incision to be mobilized bilaterally (Figure 1B). Forceps were used to pull the incision laterally to make the muscular incision about $1 \mathrm{~cm}$ away from mid-line. After blunt separation of the fat tissue, the kidney was gently exposed and the posterior renal artery was ligated under microscope (Figure $1 C, D)$. Then the ovary was dissected from the fat tissue lateral to the kidney and removed (Figure 1E, F). The remaining uterus was returned and the muscular layer was closed with a 4-0 suture. The skin incision was pulled by forceps to the contra lateral side, and the same procedure was repeated. Then the skin was closed with a 3-0 suture. After that, the rats were placed in a supine position and the right common carotid artery was ligated through a mid-line incision in the neck.

One week after the surgery, the rats were given $1 \% \mathrm{NaCl}$ in their drinking water until the designated time of sacrifice. During this period, their systolic blood pressure was measured by the tail-cuff method (Softron BP-98A) without any anesthesia.

Cerebral vascular corrosion casts were produced as reported previously. The Circle of Willis was observed under scanning electron microscope by 3 blinded inspectors. The morphological changes of branching site lesions were divided into three stages as described previously $(3,8)$. In summary, Stage 1 is characterized by a roughened apical intimal pad with irregularly shaped endothelial cell imprints. While in Stage 2 there is shallow fusiform elevation of the apical intimal pad, which is covered by abnormal endothelial cell imprints. Stage 3 indicates that a well-developed saccular aneurysm is present. We also recorded the height of the Stage 3 aneurysm, which was measured by the distance between the dome to parent artery $(7,8)$. The non-bifurcation lesions were classified as dilatation, tortuosity, or fusiform aneurysms according to the previous papers $(3,9)$.

The frozen sections of the anterior cerebral artery and olfactory artery (ACA-OA) bifurcation were obtained as described previously (1). Elastica van Gieson stain was applied following the manufacturer's guidelines (Elastica van Gieson kit, Maixin. Bio). Fluorescence stains of vascular smooth muscle were performed as described before (1). The primary antibody used was rabbit polyclonal anti-alpha smooth muscle actin antibody (1:400, Abcam) and the secondary antibody used was Alexa Fluor conjugates goat anti-rabbit IgG antibody (1:800, Cell Signaling).

Statistical analyses were performed using PASW Statistics 18.0 software. T-test or Chi-square test was used to determine any statistical differences amongst the variables between the groups. A two-sided P-value less than 0.05 indicated a statistically significant difference.

\section{RESULTS}

A comparison of the surgical characteristics of the rats in Groups 1 and 2 are shown in Table I. The surgical duration time was significantly reduced in Group 2 (55.9 vs. 38.8 minutes, $\mathrm{p}=0.02$, T-test). The need for anesthesia redose was significantly reduced in the simplified incision group $(73 \%$ vs. $27 \%, p=0.02$, Chi-Square test). For peri-surgical mortality, 3 rats in Group 1 died of redose during surgery, and another 2 died within 12 hours after surgery without any discernable cause. Only 1 rat died due to redoes in Group 2. Although fewer rats in group 2 died, there was no statistically significant difference in the mortality rate amongst the groups (33\% vs. $9 \%, p=0.147$, Chi-Square test). Post-surgical wound infection was reduced in group 2, however this difference was not statistically significant $(13 \%$ vs. $9 \%, p=0.738$, Chi-Square test).

The blood pressures of the rats in each group are shown in Figure 2. The means and standard deviations (SD) for Group 0, 1,2 and 3 were $108 \pm 13,149 \pm 16,157 \pm 15$ and $160 \pm 20 \mathrm{mmHg}$, respectively. Rats in both the multiple incision (Group1) and simplified incision (Group 2 and 3) had significantly increased blood pressure compared to the normal rats in Group 0 $(p<0.001$ for all the other groups compared with Group 0, T-test). The mean blood pressures of the rats in the simplified incision groups were slightly higher than those of rats in group 1 , but this difference did not achieve statistical significance $(p=0.264$ for comparisons between Group 2 and Group 1, 
$\mathrm{p}=0.191$ for comparisons between Group 3 and Group 1, T-test).

No aneurysms formed in the rats in Group 0. The aneurysmal morphological changes that occurred in Groups 1, 2, and 3 are shown in Table II. The majority of branching site lesions were located at the left ACA-OA bifurcation area. Even for those that formed at the left internal cerebral artery and middle cerebral artery (ICA-MCA) bifurcation, no high stage aneurysms were found. There was no statistically significant difference with regard to the incidence of Stage 2 and 3 branching site lesions between Groups 1 and 2, respectively (36\% vs. $43 \%, 18 \%$ vs. $21 \%$ ). Amongst the rats in groups 2 and 3, only the Stage 3 aneurysm significantly increased in Group 3 (61\% vs. $21 \%, p=0.031$, Chi-square test). The rats in group 3 also had significantly more Stage 3 aneurysms than the rats in Group 1 (61\% vs. $18 \%, p=0.01$, Chi-square test). The general size of the Stage 3 aneurysms in Group 3 were larger than those of Group 2 (Figure $3 \mathrm{~A}, \mathrm{~B}$ ), the mean and SD of the height of Stage 3 aneurysms in Group 3 and 2 were $93 \pm 20$ and $34 \pm 12 \mu \mathrm{m}$, respectively ( $\mathrm{p}=0.001$, T-test). Although no significant difference was observed amongst the nonbranching site lesions, the dilation of proximal segment (P1) of right posterior cerebral artery was more frequently found than the tortuosity of communicating branches of proximal anterior cerebral artery (A1). Fusiform aneurysms formed at both of these locations (Figure $3 \mathrm{C}, \mathrm{D}$ ).

Histological analysis showed a large bifurcation site aneurysm (Figure 4A-C). Special staining also confirmed discontinuing

Table I: Surgical Characteristics Between Multiple Incisions and Simplified Midline Incision

\begin{tabular}{lcc}
\hline & Group 1 (n=15) & Group 2 (n=11) \\
\hline Number of total incisions & 4 & 2 \\
Surgical duration time ${ }^{a}$ (minute, mean \pm SD) & (midabdominal, bilateral flank and neck) & (dorsal midline and neck) \\
\hline${\text { Need for anesthesia redose }{ }^{b} \text { (number of surgery) }}^{\text {Peri-surgical mortality }{ }^{c} \text { (number of rats) }}$ & $55.9 \pm 3.21$ & $38.8 \pm 3.58$ \\
\hline Post surgical wound infection (number of rats) & $11(73 \%)$ & $3(27 \%)$ \\
\hline
\end{tabular}

aSignificant difference, $p<0.001$, T-test. Data was only collected from 10 survived rats in each group.

'Significant difference, $p=0.02$, Chi-Square test.

'Peri-surgical mortality were defined as death during or with 12 hours after surgery.

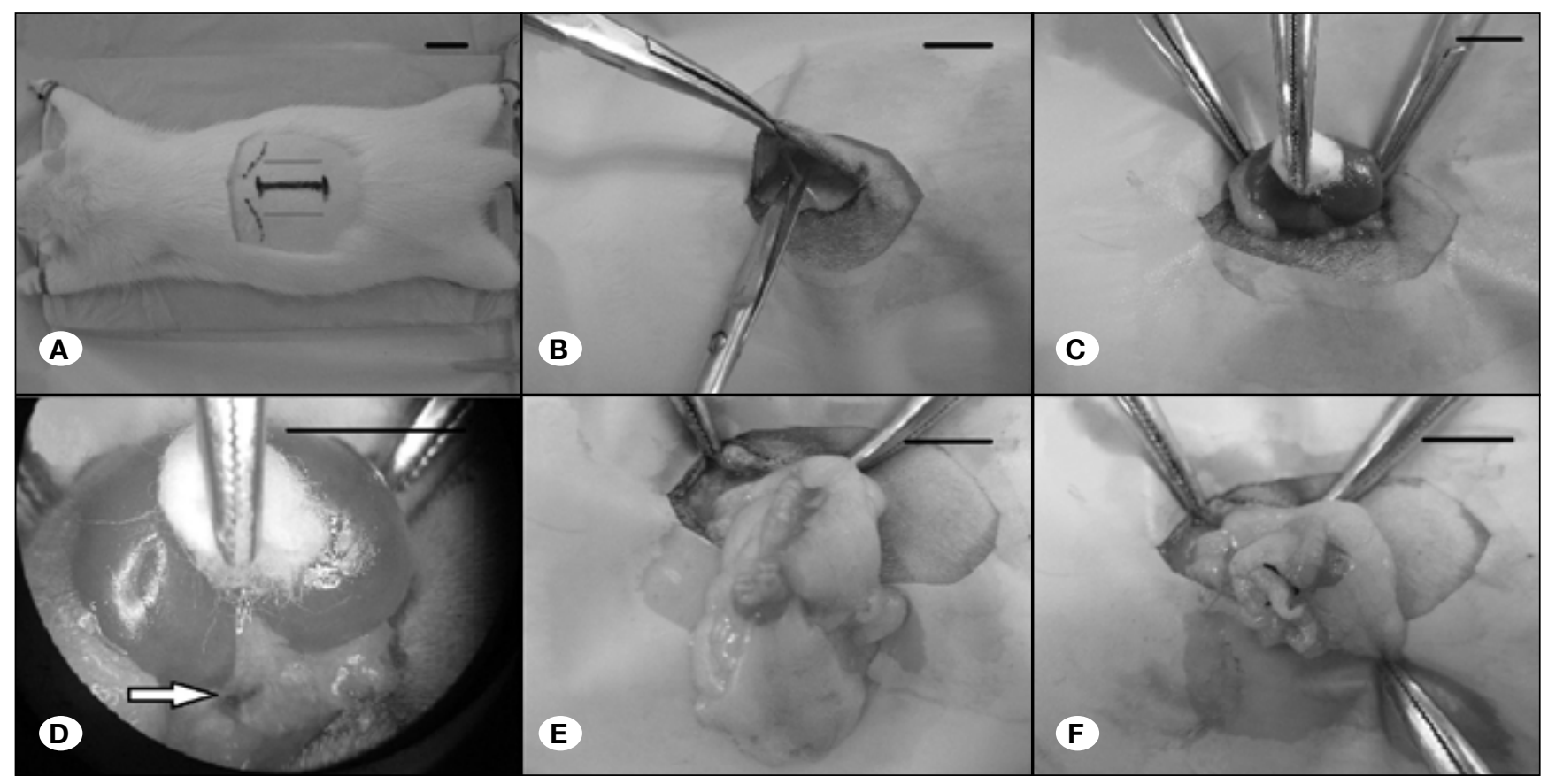

Figure 1: A) Location of skin incision (middle thick solid line) and underneath the muscle layer incisions (bilateral thin solid lines). Discontinued lines indicate costal margin. B) Dissection of the subcutaneous fascia. C) Kidney was gently exposed. D) Posterior renal artery was ligated under microscope. E, F) Ovary was removed through the same incision. Bar=1cm. 
of the internal elastic lamina and loss of the vascular smooth muscle layer in the saccular aneurysm wall; these findings have also been observed in human aneurysms (4).

\section{DISCUSSION}

In this article, we introduced a modified surgical technique to build the traditional female rat model of CA. The traditional female rat model of CA $(7,8)$ consists of a two-stage procedure in which an oophorectomy is performed 1 month after the ligation of renal and carotid arteries. Our modified technique allows all procedures to be performed in a single (one-stage) operation, consequently reducing the surgical stress placed on the experimental animals. Additionally, the rats were exposed to estrogen deficiency for a longer time. The protective effects of estrogen against aneurysms may occur by different mechanisms. For example, estrogen could enhance expression of endothelial nitric oxide synthase (17), which plays protective role for endothelial damage of aneurysm formation (16). Also, estrogen has been shown to exert antioxidant activity via the stimulation of antioxidant enzymes (13). These effects may suppress the inflammation process in the arterial wall.

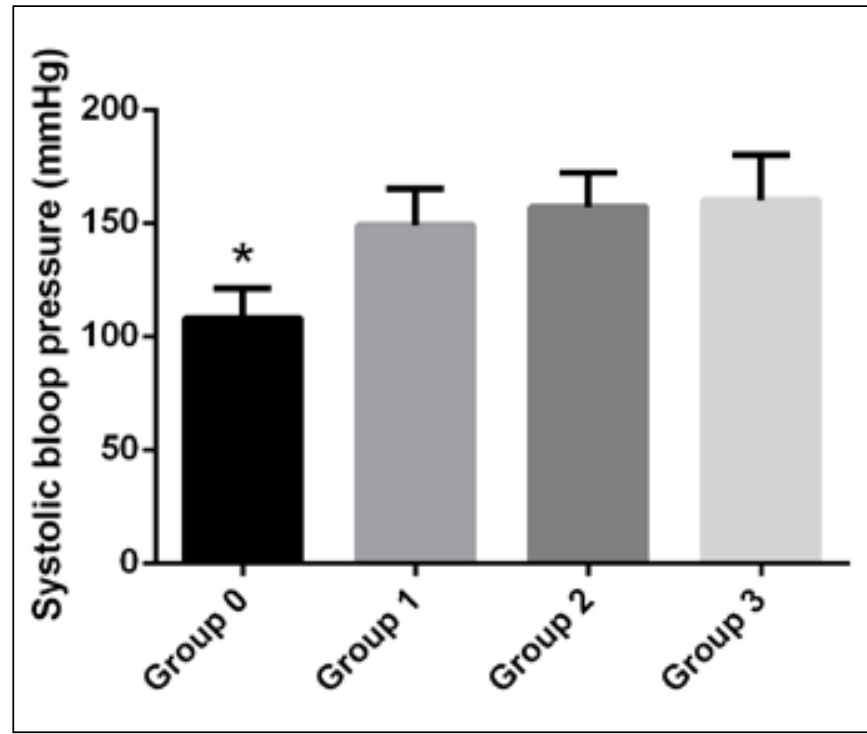

Figure 2: The systolic blood pressure stratified by groups. Data are expressed as means with standard deviation. * $p<0.05$ when groups 1, 2 and 3 were compared with group 0 . No significant difference was found in other compares between the groups ( $T$ test).

Table II: Morphological Changes Between the Groups

\begin{tabular}{|c|c|c|c|}
\hline & Group $1(n=10)$ & Group $2(n=10)$ & Group $3(n=10)$ \\
\hline Rats without any lesions & 2 & 1 & 0 \\
\hline \multicolumn{4}{|l|}{ Locations } \\
\hline \multicolumn{4}{|l|}{ Staging (ACA-OA/ICA-MCA) } \\
\hline Stage1 & $3 / 2(46 \%)$ & 3 / 2 (36\%) & $0 / 1(8 \%)$ \\
\hline Stage2 & 4 / 0 (36\%) & $5 / 1(43 \%)$ & $2 / 2(31 \%)$ \\
\hline Stage3 b & 2 / 0 (18\%) & $3 / 0(21 \%)$ & $8 / 0(61 \%)$ \\
\hline
\end{tabular}

Non-branching site lesions

\begin{tabular}{lccc}
\hline Total number of lesions & 9 & 10 & 12 \\
\hline Rats without any lesions & 1 & & 1 \\
\hline Locations & & & \\
A1-communicating & $1(11 \%)$ & $2(20 \%)$ & $3(25 \%)$ \\
Right P1 & $8(89 \%)$ & $8(80 \%)$ & $9(75 \%)$ \\
\hline Classification & & & $8(67 \%)$ \\
$\quad$ Dilatation & $5(56 \%)$ & $6(60 \%)$ & $3(25 \%)$ \\
$\quad$ Tortuosity & $3(33 \%)$ & $3(30 \%)$ & $1(8 \%)$ \\
Fusiform & $1(11 \%)$ & $1(10 \%)$ & \\
\hline
\end{tabular}

ACA: anterior cerebral artery, OA: olfactory artery, ICA: internal carotid artery, MCA: middle cerebral artery, A1: proximal segment of anterior cerebral artery, P1: proximal segment of posterior cerebral artery.

a Lesions at the $A 1$ fenestration were included in this area for analysis.

b There are significant difference in this staging distributions between Groups 2 and 3 ( $p=0.031$, Chi-square test) and Groups 1 and 3 ( $p=0.01$, Chi-square test). 


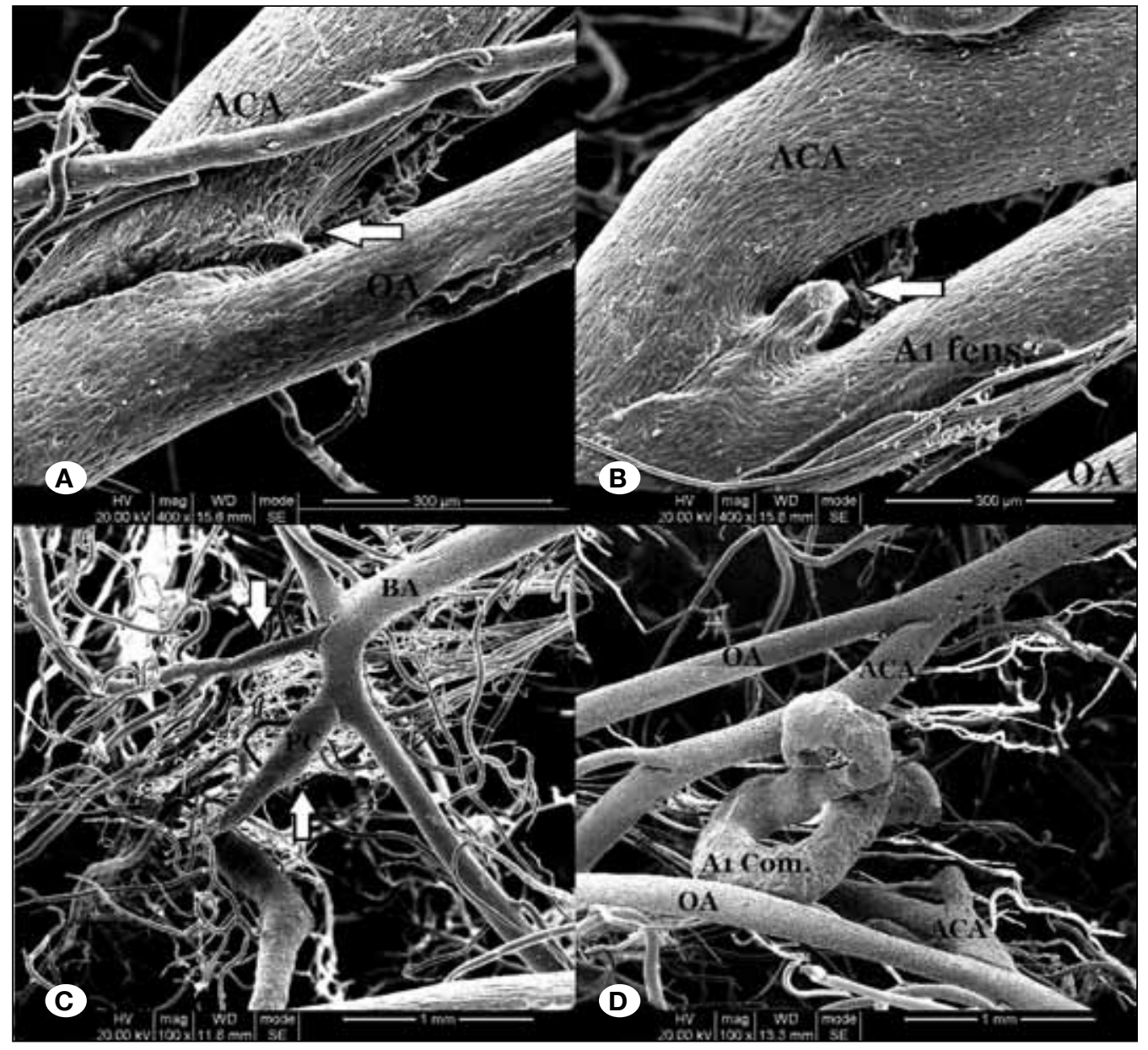

Figure 3: Representative scanning electron microscope images of cerebral vascular corrosion casts. A) Stage 3 aneurysm formation in a rat from group 2; B) Stage 3 aneurysm in a rat from group 3;

C) Dilatation and fusiform change of left posterior cerebral artery (white arrows); D) Tortuosity and fusiform changes of $A 1$ communicating artery.

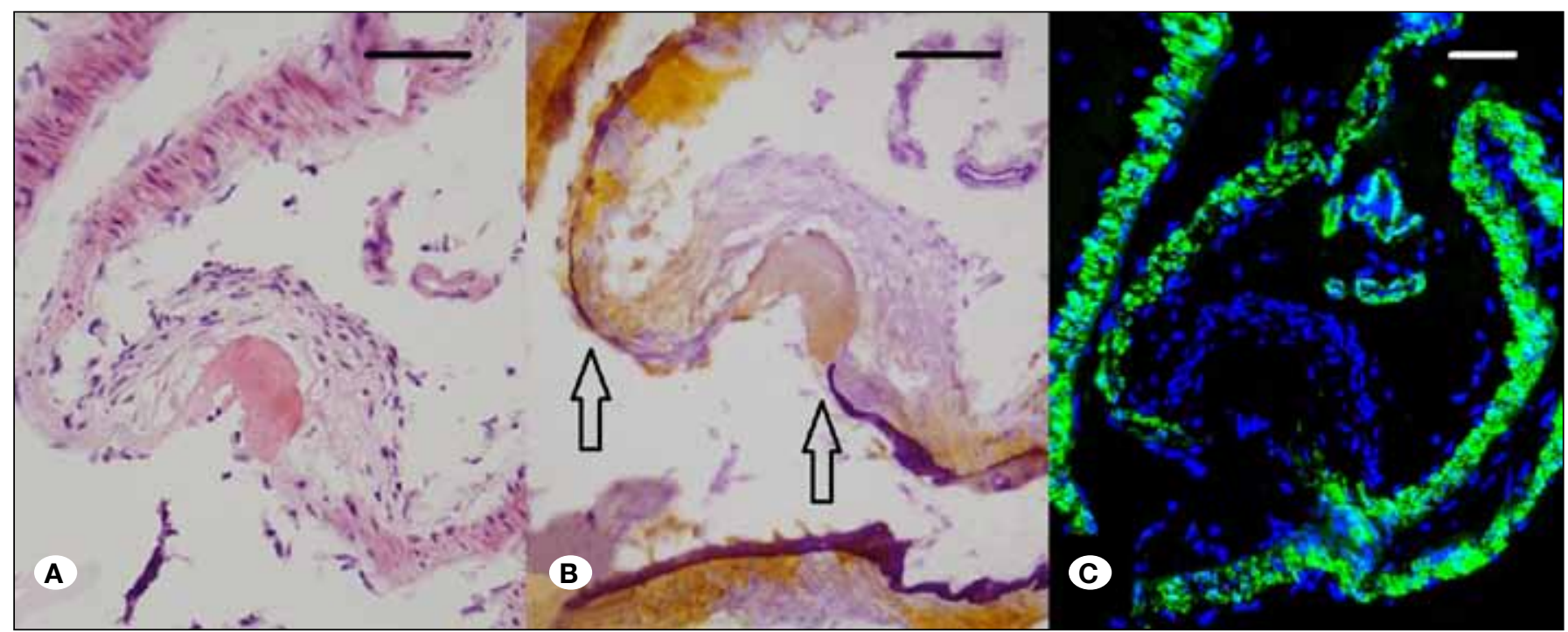

Figure 4: Histology of induced aneurysmal walls. A) Hematoxylin-Eosin stain showed abnormal protrusion with derangement wall structure at artery bifurcation. B) Elastica van Gieson stain showed discontinue of internal elastic lamina. C) Fluorescence stains showed absence of vascular smooth muscle cell at aneurysm wall, and DAPI showed cell nuclei. Bar=50 $\mu \mathrm{m}$. 
Our modified model also reduced the number of incisions required to complete all procedures as compared with the previously reported one stage method (3). In this study, we repeated the other one stage method which requires 4 incisions to complete, and compared the surgical characteristics with our model. Only 2 skin incisions were needed to perform all procedures in our method; this reduced the average operative time by 17 minutes. This time reduction decreased the need for anesthesia redose, as for anesthesia by intraperitoneal injection of ketamine $(80 \mathrm{mg} / \mathrm{kg})$ and xylazine $(8 \mathrm{mg} / \mathrm{kg})$, usually it could last for 30 to 40 minutes. Redose for rats during same surgery was risky. In our experiment, 3 rats died of redoes in Group 1, and 1 rat died of redose in Group 2. Hence the reduced mortality rate may also be related to the reduced operative time.

The technique used may also impact the incidence of postoperative wound infection. Rats have strong resistance against infection if the proper aseptic technique is applied during surgery. However rats can tear suture off by themselves or from each other after surgery. The risk of infection may be increased if the incisions are located bilaterally or on an easily reachable location such as the abdomen. In this study, compared to rats in Group 1, the infection rate was slightly reduced in the rats in Group 2. This may be due to the modified incision technique in which the incisions are placed in difficult to reach areas such as on the middle back and the neck regions. Overall, the simplified incision technique reduced the surgical stress placed on experimental rats.

The aneurysm morphology characteristics were also compared amongst the different groups. Although the simplified incision groups seem to have more advanced stage (stage 2 and 3) branching site aneurysms compared to the multiple incisions group, no statistically significant difference was found. With regard to comparison of blood pressure, there was a slight increase in blood pressure in the simplified incision group, but no statistically significant difference was observed. We postulate that the middle abdominal and bilateral flank incisions may cause more insults to the digestive system of the rat, which may compromise the high salt water intake and influence the increase in blood pressure. This may explain the slight drop in blood pressure and lower rate of advanced stage aneurysms in the multiple incision group.

Moreover, the incidence of Stage 3 saccular aneurysms increased significantly after the duration for CA formation was extended to 6 months in Group 3 rats. This indicates that CA formation progresses when continuously exposed to high hemodynamic stress. Similar results have been reported in humans; nearly $50 \%$ of the unruptured CA shows growth in 3 years (12). It also raises concerns about the proper management of unruptured CA, as the enlargement of aneurysm size is associated with a higher risk of rupture. Inspiringly, through the aneurysm rat model, several medications to suppress or reverse the progress of CA have been tested $(1,15,19)$.

Our findings also suggest that proper prolongation of the duration of aneurysm formation could induce development of a giant CA for certain research purposes. Recently, some other rodent models with use of elastase have been developed to induce large CA in a few weeks $(11,14)$. But a shorter incubation time also means a stronger insult of the pathophysiological process of aneurysm formation, which may fail to recapitulate some of the key processes in the human aneurysm progression. Additionally, elastase may influence the effects of experimental interventions. For example, a possible inhibitory effect of a medication may be masked by the strong effect of elastase between the experimental and control groups. So, the ligation model should still be considered for investigations which require large aneurysm formation, even though a longer incubation time may be needed.

For the non-branching site lesions, we found that dilation of right posterior cerebral artery was commonly observed, as it was the main collateral pathway after ligation of right common carotid artery. On the other hand, there is no obvious anterior communication artery in rats (10), so the non-branching lesions in that area were relatively rare, but a fusiform aneurysm could be formed if there are small communicating perforating arteries. These findings indicate that hemodynamic stress caused by increasing collateral flow on the whole artery could induce a fusiform aneurysm. However, there may be other factors that influence the susceptibility and progression of fusiform aneurysms, as the aneurysm formation rates amongst the rats in the 6 months group did not vary significantly from rats in the 3 months group.

\section{- CONCLUSION}

Single operation through simplified incisions resulted in a successful experimental female rat model of cerebral aneurysms. These aneurysms had the same morphological and histological characteristic as human aneurysms. Reducing the number of incisions not only saved time, but also minimized the surgical stress placed on the experimental animal. Saccular aneurysms at the branching site progressed when the duration for aneurysm formation was prolonged, and this finding could be applied when induction of large aneurysms is needed for certain research purpose. Fusiform aneurysms at the non-branching site may have an additional etiology other than hemodynamic reasons; this should be further investigated.

\section{口 REFERENCES}

1. Aoki T, Kataoka H, Ishibashi R, Nozaki K, Hashimoto $\mathrm{N}$ : Simvastatin suppresses the progression of experimentally induced cerebral aneurysms in rats. Stroke 39:1276-1285, 2008

2. Connolly ES Jr, Rabinstein AA, Carhuapoma JR, Derdeyn CP, Dion J, Higashida RT, Hoh BL, Kirkness CJ, Naidech AM, Ogilvy CS, Patel AB, Thompson BG, Vespa P; American Heart Association Stroke Council; Council on Cardiovascular Radiology and Intervention; Council on Cardiovascular Nursing; Council on Cardiovascular Surgery and Anesthesia; Council on Clinical Cardiology: Guidelines for the management of aneurysmal subarachnoid hemorrhage: A guideline for healthcare professionals from the American Heart Association/american Stroke Association. Stroke 43:1711-1737, 2012 
3. Eldawoody $H$, Shimizu H, Kimura N, Saito A, Nakayama T, Takahashi A, Tominaga T: Simplified experimental cerebral aneurysm model in rats: Comprehensive evaluation of induced aneurysms and arterial changes in the circle of Willis. Brain Res 1300:159-168, 2009

4. Hashimoto N, Handa H, Hazama F: Experimentally induced cerebral aneurysms in rats: Part III. Pathology. Surg Neurol 11:299-304, 1979

5. Hashimoto N, Handa H, Hazama F: Experimentally induced cerebral aneurysms in rats. Surg Neurol 10: 3-8, 1978

6. Hashimoto N, Hazama F: Experimentally induced cerebral aneurysms as model for non-surgical treatment. $\mathrm{J}$ Clin Neurosci 1:266-273, 1994

7. Jamous MA, Nagahiro S, Kitazato KT, Satomi J, Satoh K: Role of estrogen deficiency in the formation and progression of cerebral aneurysms. Part I: Experimental study of the effect of oophorectomy in rats. J Neurosurg 103:1046-1051, 2005

8. Jamous MA, Nagahiro S, Kitazato KT, Tamura T, Kuwayama $\mathrm{K}$, Satoh K: Role of estrogen deficiency in the formation and progression of cerebral aneurysms. Part II: Experimental study of the effects of hormone replacement therapy in rats. J Neurosurg 103:1052-1057, 2005

9. Kondo S, Hashimoto N, Kikuchi H, Hazama F, Nagata I, Kataoka $\mathrm{H}$ : Cerebral aneurysms arising at nonbranching sites. An experimental Study. Stroke 28:398-403, 1997

10. Lee RM: Morphology of cerebral arteries: Pharma Ther 66:149173, 1995

11. Nuki $\mathrm{Y}$, Tsou TL, Kurihara $C$, Kanematsu M, Kanematsu $Y$, Hashimoto T: Elastase-induced intracranial aneurysms in hypertensive mice. Hypertension 54:1337-1344, 2009
12. So TY, Dowling R, Mitchell PJ, Laidlaw J, Yan B: Risk of growth in unruptured intracranial aneurysms: A retrospective analysis. J Clin Neurosci 17:29-33, 2010

13. Strehlow K, Rotter S, Wassmann S, Adam O, Grohé C, Laufs K, Böhm M, Nickenig G: Modulation of antioxidant enzyme expression and function by estrogen. Circ Res 93:170-177, 2003

14. Tada $\mathrm{Y}$, Kanematsu $\mathrm{Y}$, Kanematsu M, Nuki Y, Liang El, Wada K, Makino $\mathrm{H}$, Hashimoto T: A mouse model of intracranial aneurysm: Technical considerations. Acta Neurochir Suppl 111:31-35, 2011

15. Tada Y, Kitazato KT, Tamura T, Yagi K, Shimada K, Kinouchi T, Satomi J, Nagahiro S: Role of mineralocorticoid receptor on experimental cerebral aneurysms in rats. Hypertension 54:552557, 2009

16. Tamura T, Jamous MA, Kitazato KT, Yagi K, Tada $Y$, Uno $M$, Nagahiro S: Endothelial damage due to impaired nitric oxide bioavailability triggers cerebral aneurysm formation in female rats. J Hypertens 27:1284-1292, 2009

17. Wagner AH, Schroeter MR, Hecker M: 17beta-estradiol inhibition of NADPH oxidase expression in human endothelial cells. FASEB J 15:2121-2130, 2001

18. Xu Y, Tian Y, Wei HJ, Dong JF, Zhang JN: Methionine diet-induced hyperhomocysteinemia accelerates cerebral aneurysm formation in rats. Neurosci Lett 494:139-144, 2011

19. Yagi K, Tada Y, Kitazato KT, Tamura T, Satomi J, Nagahiro S: Ibudilast inhibits cerebral aneurysms by downregulating inflammation-related molecules in the vascular wall of rats. Neurosurgery 66:551-559, 2010 